\title{
The role of adjuvant radiotherapy in patients with malignant phyllodes tumor of the breast: a propensity-score matching analysis
}

\author{
Wen Zhao ${ }^{1} \cdot$ Qi Tian $^{1} \cdot$ Andi Zhao $^{1} \cdot$ Biyuan Wang ${ }^{1} \cdot J_{i a o}$ Yang $^{1} \cdot$ Le Wang $^{1} \cdot$ Lingxiao Zhang $^{1} \cdot$ Danfeng Dong $^{1}$. \\ Ling Chen ${ }^{1} \cdot$ Jin Yang ${ }^{1}$ (D)
}

Received: 3 November 2019 / Accepted: 16 July 2020 / Published online: 3 August 2020

(c) The Author(s) 2020

\begin{abstract}
Background and objectives Malignant phyllodes tumor of the breast (MPTB) is a kind of rare tumor. Our objective was to investigate the role of adjuvant radiotherapy (RT) in MPTB patients.

Methods MPTB patients were identified in the Surveillance, Epidemiology and End Results (SEER) database. KaplanMeier curves and multivariable Cox proportional hazards analyses were conducted to determine the effect of adjuvant RT on MPTB patients. Propensity-score matching (PSM) method was used to balance the clinicopathological characteristics. Results A total of 1353 MPTB patients were included in our study and the median follow-up time was 99 months (range: 0-331 months). 16.7\% (226) MPTB patients received adjuvant RT, of which 49.1\% (111) received mastectomy and 50.9\% (115) underwent breast conservation surgery (BCS). Patients receiving adjuvant RT were more likely to be white, with better differentiation and larger tumors $(p<0.05)$. Multivariate analysis showed that poorer tumor differentiation grade, larger tumor size, and lymph node metastasis were associated with reduced survival while BCS was a protective factor of diseasespecific survival (DSS) (HR 0.297; 95\% CI 0.184-0.480) and overall survival (OS) (HR 0.445; 95\% CI 0.321-0.616). After PSM, survival curves showed patients did not achieve an improved OS or DSS from adjuvant RT $(p>0.05)$. In subgroup analysis, no subgroup benefited from adjuvant RT. Exploratory analysis showed a survival benefit trend from adjuvant RT in patients with tumor larger than $50 \mathrm{~mm}$ and undergoing BCS.

Conclusions Among MPTB patients, adjuvant RT did not improve OS or DSS. In patients with tumor larger than $50 \mathrm{~mm}$ and receiving BCS, a survival benefit trend from adjuvant RT existed.
\end{abstract}

Keywords MPTB $\cdot$ SEER $\cdot$ Adjuvant radiotherapy $\cdot$ PSM $\cdot$ Survival

\section{Introduction}

Phyllodes tumor of the breast (PTB) is rare, of which the incidence rate is $2 \%$ to $3 \%$ in all breast fibrous epithelial tumors, or $0.3 \%$ to $1.0 \%$ in all breast tumors $[1,2]$. It was in 1838 that Johannes Muller first reported this tumor and named it as cystosarcoma phyllodes, because of its huge neoplasia with a cystic lobulated section. Until 1981, the

Electronic supplementary material The online version of this article (https://doi.org/10.1007/s12282-020-01135-7) contains supplementary material, which is available to authorized users.

Jin Yang

1473106133@qq.com

1 Department of Medical Oncology, The First Affiliated Hospital of Xi' an Jiaotong University, No 277 Yanta West Road, Xi'an 710061, Shaanxi, People's Republic of China international histological classification group of World Health Organization (WHO) renamed it as phyllodes tumor and divided it into three subtypes: benign, borderline and malignant, according to pathological features [3, 4].

Malignant phyllodes tumor of breast (MPTB) comprises $20 \%$ of all PTB [2] and is characterized by the aggression of clinical features and propensity for local recurrence and distant metastasis [5]. It occurs most frequently in women of child-bearing stage [6] and some patients have a history of benign breast diseases, including fibroadenoma [2]. They usually present with an insidious onset and slow progression, but rapid growth in a short term. The lesions are often unilateral, single, nodular, painless masse with size varying widely, ranging from smaller than $1 \mathrm{~cm}$ to $40 \mathrm{~cm}$ [7]. As MPTB performs an unpredictable and sometimes aggressive neoplasm, it matters a lot to investigate the features of clinicopathological factors and their prognostic roles. 
Surgery is the preferred treatment for MPTB and lymph nodes resection is not recommended, as lymph node metastases are rare. The local recurrence rate is high in PTB patients, especially in MPTB patients, up to $40 \%$ of all histological types [5, 8-10]. However, the efficacy of adjuvant radiotherapy (RT) is not clear. Many investigators have focused on this topic, ending with paradoxical results [9-14]. Besides, many studies analyzed subtypes of PTB altogether or borderline and malignant PTB combined, included only a minority of malignant tumors and generally evaluated local and distant disease recurrence rate without a report on disease-specific survival (DSS) or overall survival (OS). Importantly, the imbalance in the studied population led to the interpretation of the benefit of adjuvant RT questionable.

The aim of this study was to investigate whether MPTB patients benefit from adjuvant RT through an analysis of a large cohort of well-characterized patients, identified from the Surveillance, Epidemiology and End Results (SEER) database.

\section{Materials and methods}

\section{Patients}

We obtained data from the National Cancer Institute's SEER program, which consists of 18 population-based cancer registries, between 1988 and 2015. SEER is an open-access resource for cancer-based demographic and clinical information, as well as treatment and patient survival. SEER *Stat Version 8.3.5 (http://www.seer.cancer.gov/seerstat) was used to identify eligible patients.

The inclusion criteria of MPTB patients were listed as follows: female, year of diagnosis from 1988 to 2015, breast tumor as the first and only malignant tumor diagnosis, pathologically confirmed MPTB (ICD-O-3 9020/3), unilateral tumor, surgical treatment with either mastectomy or BCS and receiving adjuvant RT or not. Demographic variables included age at diagnosis $(\leq 35,35-55$, and $>55$ years $)$, and race (white, black, and others). Tumor characteristics included laterality (left and right), T stage (T0, T1, T2, T3, and T4), N stage (N0, N1, N2, and N3), and grade (well differentiated (I), moderately differentiated (II), poorly differentiated (III) and undifferentiated (IV)). Therapies included $\mathrm{RT}$, and surgery of the primary tumor.

\section{Propensity-score matching (PSM)}

Because it was a nonrandomized and retrospective analysis, unbalanced baseline characteristics may lead to selection bias and influence the decision to administer RT. The propensity score was defined here as the probability of being assigned to surgery plus adjuvant RT cohort or surgery alone cohort was given the clinicopathological characteristics. It was estimated using the logistic regression model that had been established from the factors potentially affecting a decision of treatment modalities. These factors included age at diagnosis, race, laterality, differentiation grade, tumor size, lymph node metastasis and surgery procedure. Patients who received adjuvant $\mathrm{RT}$ were matched to other patients based on the calculated scores with an algorithm of the nearest neighbor 1:1 matching [15].

\section{Statistical analysis}

Patient demographics, tumor characteristics, and surgery procedure were compared between those who received adjuvant RT and those who did not using the Chi square test. Overall survival (OS) was used as the primary study outcome and was defined as the time from tumor diagnosis to death from any cause. Disease-specific survival (DSS) was also compared, defined as the time from tumor diagnosis to death due to MPTB. Kaplan-Meier method was used to generate survival curves and multivariate Cox proportional hazard models to identify the prognostic factors associated with DSS and OS. Hazard ratios (HRs) and 95\% confidence intervals (95\% CIs) were reported. All analyses were performed with SPSS (version 24.0; SPSS, Inc., Chicago, IL). Statistical significance was assumed at $p$ values $<0.05$.

\section{Results}

1353 MPTB patients were included in our study and the median follow-up time was 99 months (range 0-331 months). 226 (16.7\%) received adjuvant RT, while $1127(83.3 \%)$ patients did not. 51.6\% (698) were diagnosed between 35 and 55 years and the mean age at diagnosis was 46 years (range 9-96 years). Among MPTB patients with known grade information, 47.6 percent were diagnosed with a poorly differentiated or undifferentiated tumor. 43.9\% MPTB patients were diagnosed with tumor larger than $50 \mathrm{~mm}$ and the median tumor size was $46 \mathrm{~mm}$ (range $3-360 \mathrm{~mm}$ ). Only $1.3 \%$ of patients had lymph nodes metastasis. 564 (41.7\%) underwent mastectomy and 789 (58.3\%) underwent BCS. When compared with patients in the surgery alone cohort, those in the surgery plus adjuvant RT cohort were more likely to be white, with better-differentiated tumor and larger size tumor, as well as receiving mastectomy. Demographics, tumor characteristics, and therapy information of MPTB patients were shown in Table 1.

We identified the prognostic factors of MPTB patients. Poorer tumor differentiation grade, larger tumor size, and lymph node metastasis were associated with reduced DSS, and the latter two were negatively associated with OS in both univariate and multivariate Cox proportional hazard 
Table 1 The clinicopathological characteristics of MPTB patients according to the condition of adjuvant RT before PSM and after PSM

\begin{tabular}{|c|c|c|c|c|c|c|c|}
\hline \multirow[t]{3}{*}{ Variable } & \multirow{3}{*}{$\begin{array}{l}\text { All patients } \\
\text { Number (\%) }\end{array}$} & \multicolumn{3}{|l|}{ Initial cohort } & \multicolumn{3}{|l|}{ PSM cohort } \\
\hline & & Surgery alone & Surgery + RT & $P$ value & Surgery alone & Surgery + RT & $P$ value \\
\hline & & Number (\%) & Number (\%) & & Number (\%) & Number (\%) & \\
\hline Age (years) & & & & 0.450 & & & 0.925 \\
\hline$\leq 35$ & $177(13.1)$ & $151(13.4)$ & $26(11.5)$ & & $27(12.2)$ & $26(11.5)$ & \\
\hline $35-55$ & $698(51.6)$ & $573(50.8)$ & $125(55.3)$ & & $125(56.3)$ & $125(55.3)$ & \\
\hline$>55$ & $478(35.3)$ & $403(35.8)$ & $75(33.2)$ & & $70(31.5)$ & $75(33.2)$ & \\
\hline Race & & & & 0.030 & & & 0.114 \\
\hline White & 995 (73.5) & $813(72.1)$ & $182(80.5)$ & & $192(86.5)$ & $182(80.5)$ & \\
\hline Black & $150(11.1)$ & $130(11.5)$ & $20(8.8)$ & & $18(8.1)$ & $20(8.8)$ & \\
\hline Others and unknown & $208(15.4)$ & $184(16.3)$ & $24(10.6)$ & & $12(5.4)$ & $24(10.6)$ & \\
\hline Laterality & & & & 0.047 & & & 0.942 \\
\hline Left & $655(48.4)$ & $532(47.2)$ & $123(54.4)$ & & $120(54.1)$ & $123(54.4)$ & \\
\hline Right & $698(51.6)$ & $595(52.8)$ & $103(45.6)$ & & $102(45.9)$ & $106(45.6)$ & \\
\hline Grade & & & & $<0.001$ & & & 0.948 \\
\hline I & $162(12.0)$ & $118(10.5)$ & $44(19.5)$ & & $42(18.5)$ & 44 (19.5) & \\
\hline II & $199(14.7)$ & 165 (14.6) & $34(15.0)$ & & $30(13.5)$ & $34(15.0)$ & \\
\hline $\mathrm{III}+\mathrm{IV}$ & $328(24.2)$ & $291(25.9)$ & $37(16.4)$ & & $37(16.7)$ & $37(16.4)$ & \\
\hline Unknown & $664(49.1)$ & $553(49.1)$ & $111(49.1)$ & & $114(51.4)$ & $111(49.1)$ & \\
\hline Tumor size(mm) & & & & $<0.001$ & & & 0.416 \\
\hline$\leq 50$ & $759(56.1)$ & $668(59.3)$ & $91(40.3)$ & & 99 (44.6) & $91(40.3)$ & \\
\hline $50-100$ & $364(26.9)$ & $294(26.1)$ & $70(31.0)$ & & $71(32.0)$ & $70(31.0)$ & \\
\hline$>100$ & $230(17.0)$ & $165(14.6)$ & $65(28.8)$ & & $52(23.4)$ & $65(28.8)$ & \\
\hline Lymph node & & & & 0.935 & & & 0.248 \\
\hline Negative & 1336 (98.7) & $1113(98.8)$ & $223(98.7)$ & & $0(0.0)$ & $3(1.3)$ & \\
\hline Positive & $17(1.3)$ & $14(1.2)$ & $3(1.3)$ & & $226(100.0)$ & $223(98.7)$ & \\
\hline Surgery procedure & & & & 0.013 & & & 0.388 \\
\hline Mastectomy & $564(41.7)$ & $453(40.2)$ & $111(49.1)$ & & $100(45.0)$ & $111(49.1)$ & \\
\hline BCS & $789(58.3)$ & $674(59.8)$ & $115(50.9)$ & & $122(55.0)$ & $115(50.9)$ & \\
\hline
\end{tabular}

$R T$ radiotherapy, $M P T B$ malignant phyllodes tumor of the breast, $P S M$ propensity-score matching, Grade I well differentiated; II moderately differentiated; III poorly differentiated; IV undifferentiated, $B C S$ breast conservation surgery

models (Table 2) while BCS was a protective factor of DSS (HR 0.297; 95\% CI 0.184-0.480) and OS (HR 0.445; 95\% CI 0.321-0.616). However, we didn't find the protective role of adjuvant RT in MPTB patients. The detailed information on risk factors was listed in Table 2.

Before PSM, Kaplan-Meier curves showed no significant difference in DSS and OS between the surgery alone cohort and the surgery plus adjuvant RT cohort $(p=0.264$ and $p=0.581$, respectively) (Fig. 1). To exclude the effect of clinicopathological characteristics differences between the surgery only cohort and the surgery plus adjuvant RT cohort, we used the PSM method to balance the characteristics differences. After the PSM, the characteristics of the two cohorts were balanced (Table 1). However, there was no significant difference no matter in DSS or OS (Fig. 2).

We tried to identify a subgroup of MPTB patients achieving survival benefits from adjuvant RT. However, it failed and no subgroup was observed benefited from adjuvant RT
(Figs. 3 and 4). As demonstrated above, tumor size was an important prognostic factor of DSS and OS, and MPTB patients with tumor of $50-100 \mathrm{~mm}$ or $>100 \mathrm{~mm}$ had worse DSS and OS (Table 2). The role of adjuvant RT in MPTB patients with tumor $>50 \mathrm{~mm}$ was investigated, stratified by surgery procedure. Interestingly, there was a trend that patients with tumor $>50 \mathrm{~mm}$ benefited from adjuvant RT if they received BCS, though it wasn't statistically significant (Supplementary Fig. 2). However, a similar trend was not observed in those receiving mastectomy (Supplementary Fig. 3).

\section{Discussion}

PTB is rare and the pathological diagnosis criteria are complex. According to mitotic figures, stromal cell atypia, tumor borders, stromal cell hypercellularity and overgrowth, PTB 
Table 2 Prognostic factors for DSS and OS in MPTB patients using univariate COX analysis model and multivariate COX analysis model

\begin{tabular}{|c|c|c|c|c|c|c|c|c|}
\hline \multirow[t]{3}{*}{ Variable } & \multicolumn{4}{|c|}{ Disease specific survival } & \multicolumn{4}{|c|}{ Overall survival } \\
\hline & \multicolumn{2}{|c|}{ Univariate analysis } & \multicolumn{2}{|c|}{ Multivariate analysis } & \multicolumn{2}{|c|}{ Univariate analysis } & \multicolumn{2}{|c|}{ Multivariate analysis } \\
\hline & HR & $95 \% \mathrm{CI}$ & HR & $95 \% \mathrm{CI}$ & HR & $95 \% \mathrm{CI}$ & HR & $95 \% \mathrm{CI}$ \\
\hline \multicolumn{9}{|l|}{ Age (years) } \\
\hline$\leq 35$ & Reference & & Reference & & Reference & & Reference & \\
\hline $35-55$ & 1.485 & $0.784-2.811$ & 1.387 & $0.728-2.641$ & 0.796 & $0.541-1.171$ & 0.789 & $0.535-1.165$ \\
\hline$>55$ & 1.352 & $0.695-2.630$ & 1.327 & $0.675-2.609$ & 0.768 & $0.509-1.160$ & 0.837 & $0.549-1.275$ \\
\hline \multicolumn{9}{|l|}{ Race } \\
\hline White & Reference & & Reference & & Reference & & Reference & \\
\hline Black & 1.390 & $0.830-2.328$ & 0.871 & $0.491-1.545$ & 1.896 & $1.351-2.663$ & 1.363 & $0.936-1.986$ \\
\hline Others and unknown & 1.365 & $0.861-2.164$ & 0.990 & $0.581-1.686$ & 1.172 & $0.816-1.682$ & 1.020 & $0.679-1.533$ \\
\hline \multicolumn{9}{|l|}{ Laterality } \\
\hline Left & Reference & & Reference & & Reference & & Reference & \\
\hline Right & 0.868 & $0.605-1.245$ & 0.827 & $0.574-1.193$ & 0.825 & $0.628-1.082$ & 0.783 & $0.594-1.032$ \\
\hline \multicolumn{9}{|l|}{ Grade } \\
\hline I & Reference & & Reference & & Reference & & Reference & \\
\hline II & 0.490 & $0.251-0.958$ & 0.981 & $0.451-1.868$ & 0.412 & $0.228-0.754$ & 0.670 & $0.361-1.246$ \\
\hline $\mathrm{III}+\mathrm{IV}$ & 0.648 & $0.373-1.125$ & 1.966 & $1.076-3.579$ & 0.709 & $0.444-1.134$ & 1.747 & $1.037-2.943$ \\
\hline Unknown & 0.458 & $0.272-0.772$ & 1.387 & $0.758-2.538$ & 0.596 & $0.382-0.929$ & 1.155 & $0.705-1.892$ \\
\hline \multicolumn{9}{|l|}{ Tumor size(mm) } \\
\hline$\leq 50$ & Reference & & Reference & & Reference & & Reference & \\
\hline $50-100$ & 2.828 & $1.747-4.579$ & 2.373 & $1.425-3.953$ & 1.901 & $1.361-2.654$ & 1.758 & $1.216-2.541$ \\
\hline$>100$ & 7.238 & $4.606-11.374$ & 4.494 & $2.590-7.796$ & 4.111 & $2.969-5.694$ & 2.921 & $1.938-4.404$ \\
\hline \multicolumn{9}{|l|}{ Lymph node } \\
\hline Negative & Reference & & Reference & & Reference & & Reference & \\
\hline Positive & 9.746 & $4.741-20.035$ & 4.159 & $1.735-9.966$ & 7.712 & $3.935-15.114$ & 4.152 & $1.912-9.019$ \\
\hline \multicolumn{9}{|l|}{ Surgery procedure } \\
\hline Mastectomy & Reference & & Reference & & Reference & & Reference & \\
\hline $\mathrm{BCS}$ & 0.188 & $0.121-0.290$ & 0.297 & $0.184-0.480$ & 0.333 & $0.249-0.446$ & 0.445 & $0.321-0.616$ \\
\hline \multicolumn{9}{|l|}{ Radiotherapy } \\
\hline No & Reference & & Reference & & Reference & & Reference & \\
\hline Yes & 1.339 & $0.842-2.130$ & 0.947 & $0.589-1.522$ & 1.202 & $0.818-1.765$ & 0.908 & $0.613-1.344$ \\
\hline
\end{tabular}

$D S S$ disease-specific survival, $O S$ overall survival, $M P T B$ malignant phyllodes tumor of the breast, $H R$ hazard ratio, $C I$ confidence interval, Grade I well differentiated; II moderately differentiated; III poorly differentiated; IV undifferentiated, $B C S$ breast conservation surgery

can be divided into three subtypes [3, 4], of which 35-64\% are benign PTB, and the rest are divided into borderline and malignant subtype $[11,16]$. It has been reported that about 500 women are diagnosed with MPTB every year in the United States. For MPTB, the tumor often showed infiltrative growth, carried with an unclear tumor border, infiltrated the surrounding tissue; stromal cells showed significant overgrowth and obvious atypia, sometimes accompanied by heterologous differentiation; mitotic activity was $\geq 10$ mitotic figures/10 HPF, and bleeding and necrosis occurred in large areas. Compared with the other two subtypes, MPTB is characterized by a higher risk of local recurrence and distant metastasis [5]. The extensive pathological features of MPTB pose difficulties to the preoperative diagnosis and do not reliably predict clinical behaviors. Besides, specific parameters predictive of recurrence and survival have not been established. Having a good knowledge of the clinical characteristics in MPTB not only indicates prognosis but also helps diagnosis.

In our analysis, more than half of the patients were diagnosed at 35-55 years, consistent with previous reports [17]. About half of the MPTB patients with known differentiation grade carried tumor of poorly differentiated or undifferentiated, associated with reduced DSS and OS. Different from breast tumor of other histology significantly, 43.9\% MPTB patients were diagnosed with tumors larger than $50 \mathrm{~mm}$, in line with previous studies [1, 10, 11, 13, 18, 19]; and large tumor size impaired DSS and OS. Only 17 (1.3\%) patients were found with lymph node metastasis in our analysis. Previous investigations also 

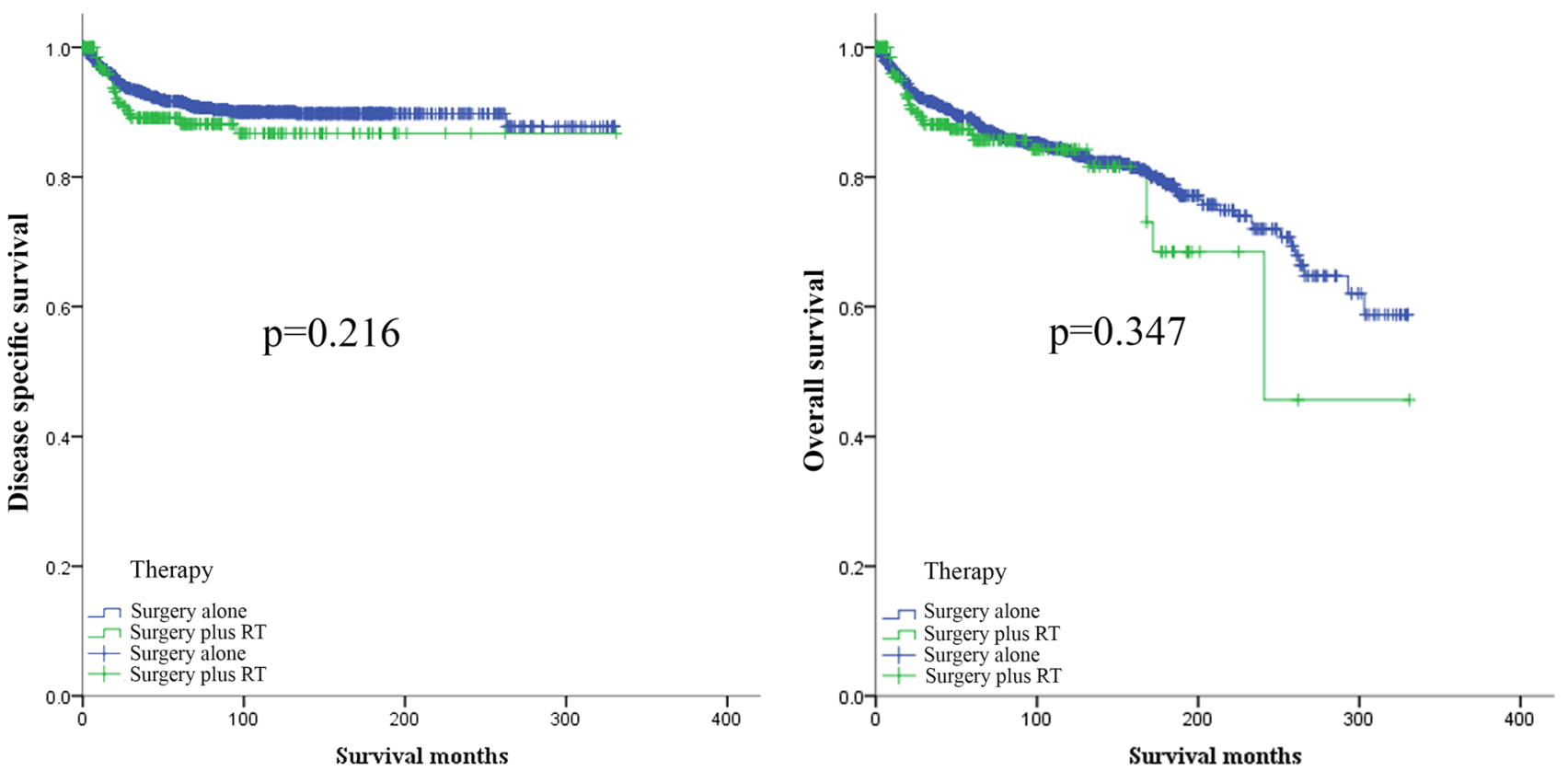

Fig. 1 The effect of adjuvant radiotherapy in MPTB patients before PSM. MPTB malignant phyllodes tumor of breast, PSM propensity-sore matching, $R T$ radiotherapy
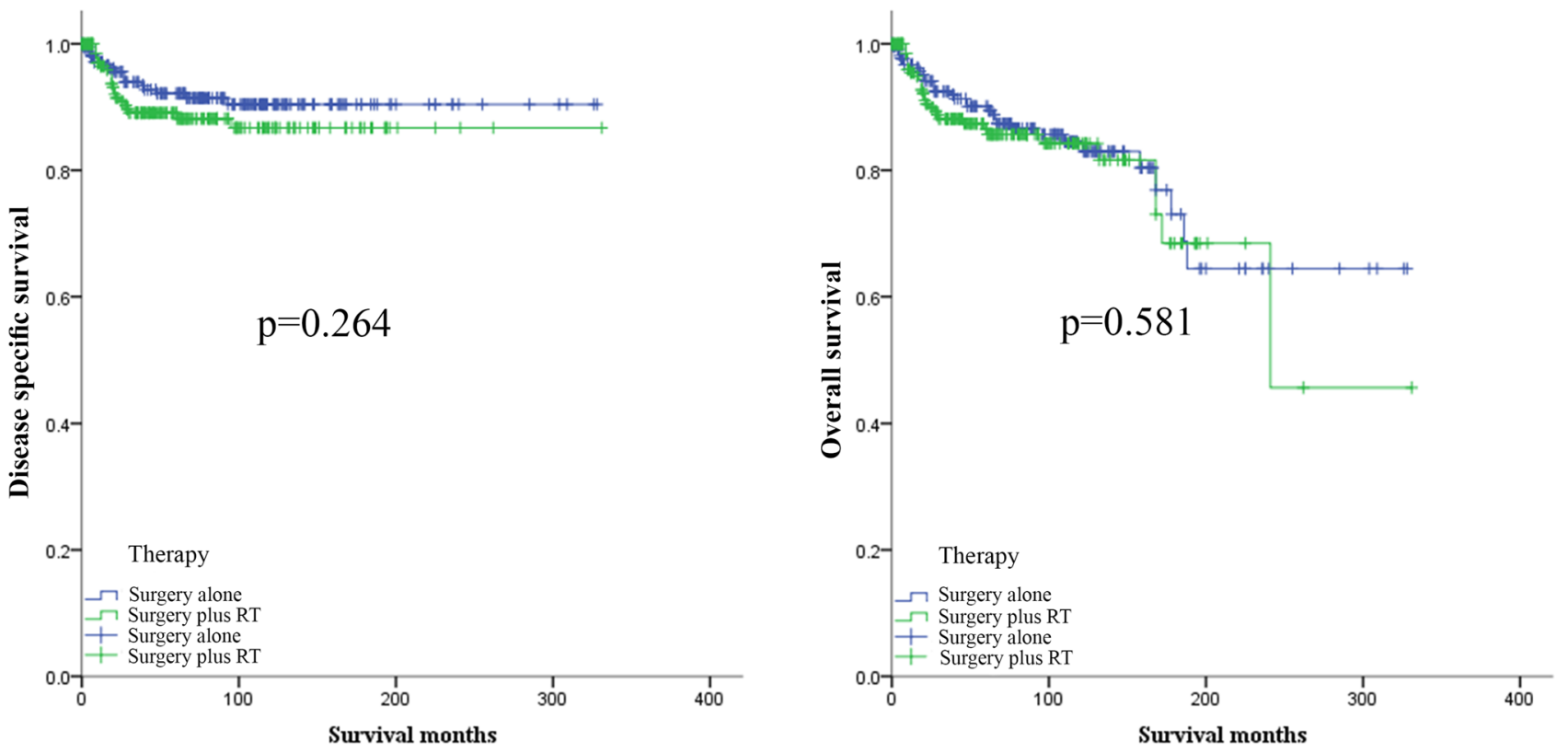

Fig. 2 The effect of adjuvant radiotherapy in MPTB patients after PSM. MPTB malignant phyllodes tumor of breast, PSM propensity-sore matching, $R T$ radiotherapy

showed a low incidence of lymph node metastasis among MPTB patients [20-24]. Surgical resection is the preferred treatment. Previous studies demonstrated mastectomy cannot provide a benefit in DSS compared with BCS in MPTB patients [9, 25]. In our analysis, Kaplan-Meier survival curves showed improved survival in patients undergoing
BCS than those undergoing mastectomy no matter receiving adjuvant RT or not $(p<0.001)$ (Supplementary Fig. 1), but patients receiving mastectomy tended to carry more risk factors (Supplementary 1). Besides, multivariate Cox analysis showed that BCS did improve survival. Therefore, 


\section{Charcateristics}

\section{HR(95\%CI)}

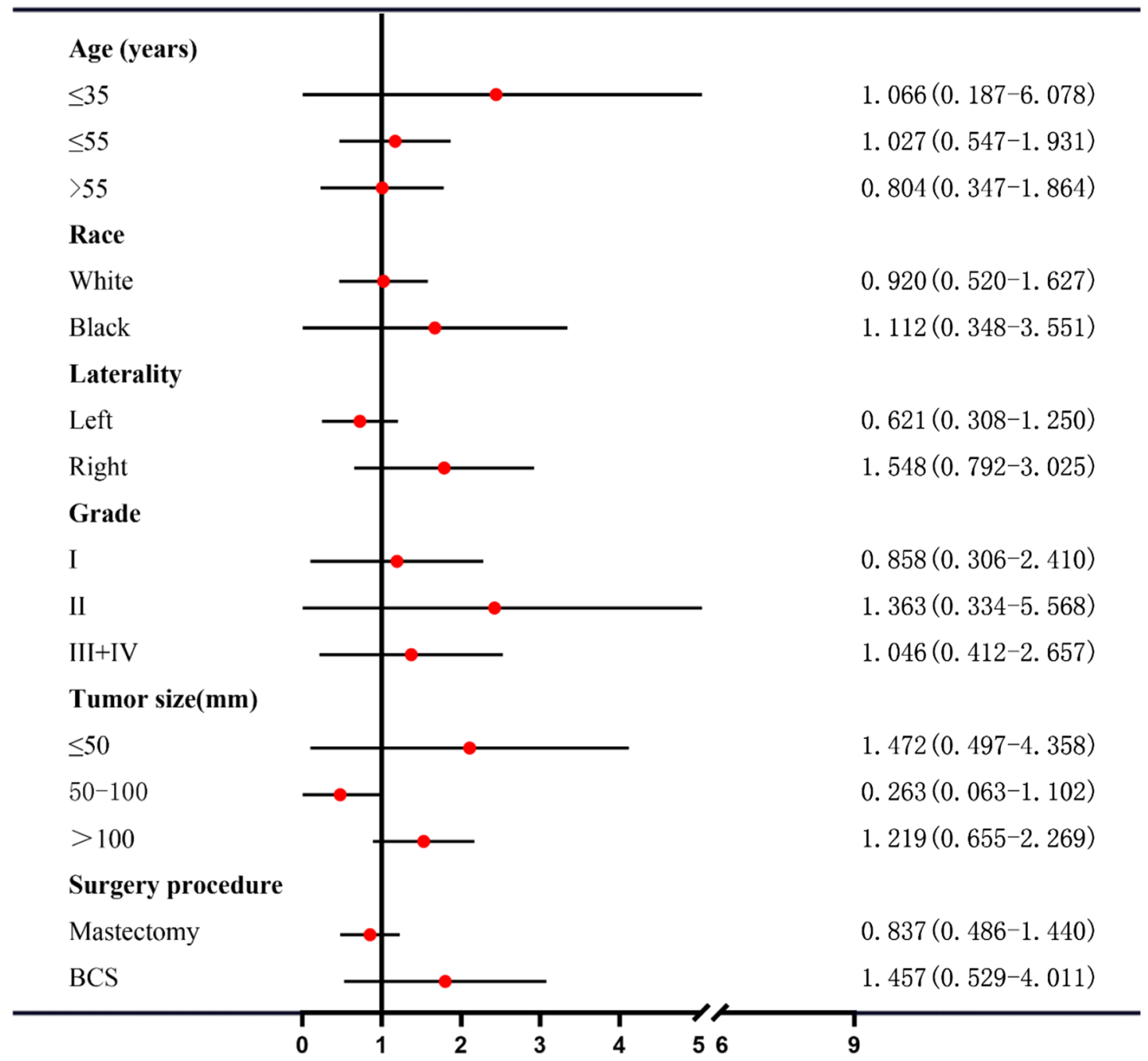

Fig. 3 Subgroup analysis to identify the effect of adjuvant radiotherapy on BCSS of MPTB patients. BCSS breast cancer-specific survival, $M P T B$ malignant phyllodes tumor of breast

surgeons should give priority to BCS in the context of a good cosmetic and oncologic outcome for MPTB patients.

Despite the complete surgical resection, the local failure rate is still high; and the local recurrence rate is up to $40 \%$ in all PTB patients $[5,8-10]$. To control the high local recurrence, some studies have investigated the role of adjuvant therapy and most focus was put on postoperative radiation therapy. The study conducted by Gnerlich et al. was the largest analysis investigating the role of adjuvant RT on MPTB patients [14]. It included 3,120 patients with MPTB, of which $14.3 \%$ of women received adjuvant RT. They found that adjuvant RT significantly reduced local recurrence (adjusted HR 0.43, 95\% CI 0.19-0.95). In other retrospective studies, local control rate of patients receiving adjuvant RT was higher when compared with those only undergoing surgery, but the characteristics between groups were uneven significantly [26, 27]. Up to now, there only existed one prospective trial. However, they treated borderline and malignant PTB patients combined, of which thirty patients (65\%) had MPTB [12]. All the patients received BCS with negative margins and received adjuvant RT subsequently. All the 46 patients didn't develop a local recurrence and it showed that margin-negative surgery combined adjuvant RT was a very effective therapy for borderline and malignant PTB in controlling local recurrence. These studies indicated that adjuvant RT improved the local control rates in MPTB patients.

Although the high local control rate achieved from adjuvant RT, whether this effect could translate into survival benefit remains controversial. The largest analysis 


\section{Charcateristics}

\section{$\operatorname{HR}(95 \% \mathrm{CI})$}

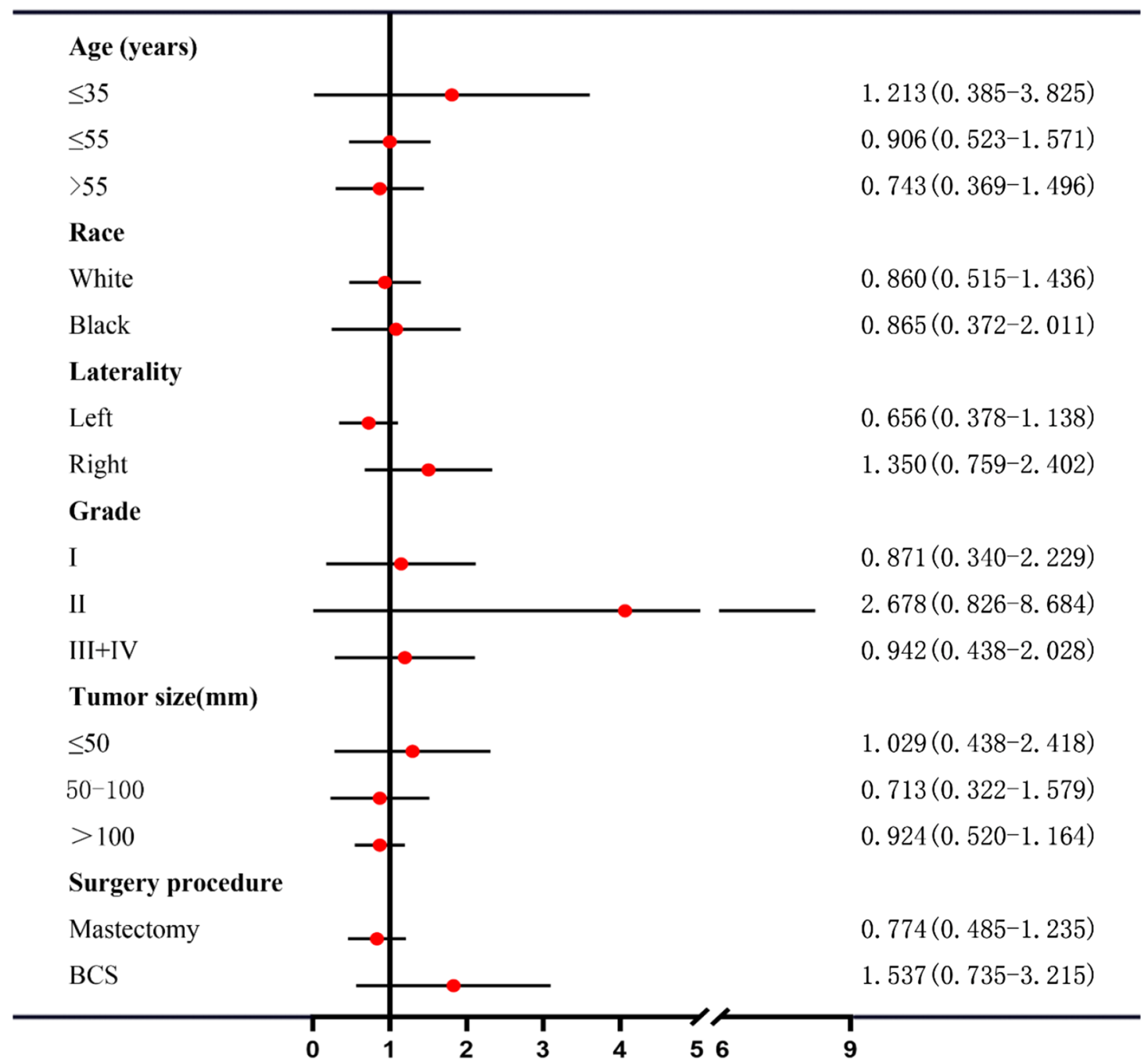

Fig. 4 Subgroup analysis to identify the effect of adjuvant radiotherapy on OS of MPTB patients. OS overall survival, MPTB malignant phyllodes tumor of breast

conducted by Gnerlich et al. showed that adjuvant RT had no effect on disease-free survival or OS [14]. However, Pandey et al. found that PTB patients undergoing adjuvant RT showed improved 5-year disease-free survival compared with those who didn't (61\% vs. $25 \%$ ), but it did not achieve a statistically significant difference $(p=0.16)$ [28]. Study conducted by Macdonald OK et al. included MPTB patients diagnosed at 1983-2002 registering in SEER database. They found that adjuvant RT predicted for worse DSS when implemented compared with surgery alone [9]. Another study conducted by Kim YJ and Kim $\mathrm{K}$ also included patients registering in the SEER database. They extended accrual period and enrolled patients diagnosed at 1983-2013. It indicated that adjuvant RT group were not inferior to the non-RT group on DSS [28]. In this retrospective analysis, the distribution of clinicopathological factors is uneven; adjuvant RT group had more adverse features such as high grade, large size, and advanced tumor extension compared with the non-RT group. In our study, we adopted the PSM method to balance the clinicopathological characteristic differences. Propensity score was estimated using the logistic regression model established from the factors potentially affecting a decision of treatment modalities, including age at diagnosis, race, laterality, differentiation grade, tumor size, lymph node metastasis and surgery procedure. Patients receiving adjuvant RT were matched to other patients based on the calculated scores with an algorithm of the nearest neighbor 1:1 matching. Table 1 showed that there existed no significant differences between the surgery plus adjuvant 
RT cohort and the surgery alone cohort. Survival curves showed no DSS or OS benefit from adjuvant RT among MPTB patients.

We next tried to investigate whether there existed any subgroup that would benefit from adjuvant RT. Pezner et al. included 478 PTB patients receiving treatment from 1964 to 2005 in the IMPAC National Oncology Database [29]. Five-year local control rates for patients with $0-2 \mathrm{~cm}$, $2-5 \mathrm{~cm}$ and $5-10 \mathrm{~cm}$ tumors and undergoing lumpectomy were $91 \%, 85 \%$, and $59 \%$, respectively. These results indicated large tumor size associated with reduced recurrence-free survival. Besides, large tumor size was negatively correlated with DSS and OS in our analysis. BCS is an appropriate treatment for MPTB patients if a good cosmetic and oncologic outcome are feasible [9] and was identified as a protective factor. However, the large average presenting tumor size of MPTB [1, 10, 11, 13, 18] limit the surgeon's ability to achieve negative margins with BCS alone. Considering all these factors, we next investigated the role of adjuvant RT in patients with tumor $>50 \mathrm{~mm}$ stratified by surgery procedure. We found that in MPTB patients with tumor $>50 \mathrm{~mm}$ and receiving BCS, they achieved DSS and OS benefits from adjuvant RT though there were no statistical differences. By contrast, there existed no DSS and OS benefit in patients receiving mastectomy. Our results reminded clinicians considering adjuvant RT in MPTB patients with tumor $>50 \mathrm{~mm}$ and receiving $\mathrm{BCS}$; those receiving mastectomy may be free of postoperative radiation therapy.

We would like to acknowledge the limitations of our study. Because of the nature of retrospective analyses, we could not exclude selection bias. However, multivariate analyses and PSM method were employed to reduce potential confounding factors. Notably, some variables that play important prognostic roles among MPTB patients are not available in the SEER database, including tumor margin status, radiation dosage, and other histology factors. Finally, we failed to include local recurrence data in our study because of the absence of information in the SEER database. As one of the largest population-based analysis to date, these results indicated that adjuvant RT did not improve DSS or OS in MPTB patients.

\section{Compliance with ethical standards}

Conflict of interest All authors declare that they have no conflicts of interest.

Ethical approval All procedures performed in studies involving human participants were in accordance with the ethical standards of the institutional and/or national research committee and with the 1964 Helsinki declaration and its later amendments or comparable ethical standards. For this type of study formal consent is not required.
Open Access This article is licensed under a Creative Commons Attribution 4.0 International License, which permits use, sharing, adaptation, distribution and reproduction in any medium or format, as long as you give appropriate credit to the original author(s) and the source, provide a link to the Creative Commons licence, and indicate if changes were made. The images or other third party material in this article are included in the article's Creative Commons licence, unless indicated otherwise in a credit line to the material. If material is not included in the article's Creative Commons licence and your intended use is not permitted by statutory regulation or exceeds the permitted use, you will need to obtain permission directly from the copyright holder. To view a copy of this licence, visit http://creativecommons.org/licenses/by/4.0/.

\section{References}

1. Guerrero MA, Ballard BR, Grau AM. Malignant phyllodes tumor of the breast: review of the literature and case report of stromal overgrowth. Surg Oncol. 2003;12(1):27-37.

2. Zhou ZR, et al. Phyllodes tumors of the breast: diagnosis, treatment and prognostic factors related to recurrence. J Thorac Dis. 2016;8(11):3361-8.

3. Tan PH, et al. Phyllodes tumors of the breast: the role of pathologic parameters. Am J Clin Pathol. 2005;123(4):529-40.

4. The World Health Organization. Histological typing of breast tumors. Neoplasma. 1983;30(1):113-23.

5. Parker SJ, Harries SA. Phyllodes tumours. Postgrad Med J. 2001;77(909):428-35.

6. Guillot E, et al. Management of phyllodes breast tumors. Breast J. 2011;17(2):129-37.

7. Hawkins RE, et al. The clinical and histologic criteria that predict metastases from cystosarcoma phyllodes. Cancer. 1992;69(1):141-7.

8. Asoglu $\mathrm{O}$, et al. Risk factors for recurrence and death after primary surgical treatment of malignant phyllodes tumors. Ann Surg Oncol. 2004;11(11):1011-7.

9. Macdonald OK, et al. Malignant phyllodes tumor of the female breast: association of primary therapy with cause-specific survival from the Surveillance, Epidemiology, and End Results (SEER) program. Cancer. 2006;107(9):2127-33.

10. Belkacemi Y, et al. Phyllodes tumor of the breast. Int J Radiat Oncol Biol Phys. 2008;70(2):492-500.

11. Reinfuss M, et al. The treatment and prognosis of patients with phyllodes tumor of the breast: an analysis of 170 cases. Cancer. 1996;77(5):910-6.

12. Barth RJ Jr, et al. A prospective, multi-institutional study of adjuvant radiotherapy after resection of malignant phyllodes tumors. Ann Surg Oncol. 2009;16(8):2288-94.

13. Chaney AW, et al. Primary treatment of cystosarcoma phyllodes of the breast. Cancer. 2000;89(7):1502-11.

14. Gnerlich JL, et al. Utilization of radiotherapy for malignant phyllodes tumors: analysis of the National Cancer Data Base, 19982009. Ann Surg Oncol. 2014;21(4):1222-30.

15. Austin PC. An introduction to propensity score methods for reducing the effects of confounding in observational studies. Multivar Behav Res. 2011;46(3):399-424.

16. Salvadori B, et al. Surgical treatment of phyllodes tumors of the breast. Cancer. 1989;63(12):2532-6.

17. Khosravi-Shahi P. Management of non metastatic phyllodes tumors of the breast: review of the literature. Surg Oncol. 2011;20(4):e143-8.

18. Barrio AV, et al. Clinicopathologic features and long-term outcomes of 293 phyllodes tumors of the breast. Ann Surg Oncol. 2007;14(10):2961-70. 
19. Varghese SS, et al. Radiotherapy in phyllodes tumour. J Clin Diagn Res. 2017;11(1):xc01-3.

20. Telli ML, et al. Phyllodes tumors of the breast: natural history, diagnosis, and treatment. J Natl Compr Canc Netw. 2007;5(3):324-30.

21. Chen WH, et al. Surgical treatment of phyllodes tumors of the breast: retrospective review of 172 cases. J Surg Oncol. 2005;91(3):185-94.

22. Reinfuss M, Mitus J, Stelmach A. Phyllodes tumor of the breast. Strahlenther Onkol. 1995;171(1):5-11.

23. Mishra SP, et al. Phyllodes tumor of breast: a review article. ISRN Surg. 2013;2013:361469.

24. Spitaleri G, et al. Breast phyllodes tumor: a review of literature and a single center retrospective series analysis. Crit Rev Oncol Hematol. 2013;88(2):427-36.

25. Zhou ZR, et al. Prognostic factors in breast phyllodes tumors: a nomogram based on a retrospective cohort study of 404 patients. Cancer Med. 2018;7(4):1030-42.
26. $\mathrm{Xu} \mathrm{B}$, et al. National consensus in China on diagnosis and treatment of patients with advanced breast cancer. Ann Transl Med. 2015;3(17):242.

27. McGale P, et al. Effect of radiotherapy after mastectomy and axillary surgery on 10-year recurrence and 20 -year breast cancer mortality: meta-analysis of individual patient data for 8135 women in 22 randomised trials. Lancet. 2014;383(9935):2127-35.

28. Kim YJ, Kim K. Radiation therapy for malignant phyllodes tumor of the breast: an analysis of SEER data. Breast. 2017;32:26-32.

29. Pezner RD, Schultheiss TE, Paz IB. Malignant phyllodes tumor of the breast: local control rates with surgery alone. Int J Radiat Oncol Biol Phys. 2008;71(3):710-3.

Publisher's Note Springer Nature remains neutral with regard to jurisdictional claims in published maps and institutional affiliations. 